\title{
Manuels de français langue étrangère et pratiques des enseignants
}

\section{Margaret Bento}

\section{(2) OpenEdition}

1 Journals

\section{Édition électronique}

URL : http://journals.openedition.org/rdlc/739

DOI : $10.4000 /$ rdlc.739

ISSN : $1958-5772$

Éditeur

ACEDLE

\section{Référence électronique}

Margaret Bento, "Manuels de français langue étrangère et pratiques des enseignants », Recherches en didactique des langues et des cultures [En ligne], 12-2 | 2015, mis en ligne le 20 novembre 2015

consulté le 10 décembre 2020. URL : http://journals.openedition.org/rdlc/739; DOI : https://doi.org/ $10.4000 /$ rdlc.739

Ce document a été généré automatiquement le 10 décembre 2020.

\section{c) (i)}

Recherches en didactique des langues et des cultures is licensed under a Creative Commons AttributionNonCommercial-NoDerivatives 4.0 International License 


\title{
Manuels de français langue étrangère et pratiques des enseignants
}

\author{
Margaret Bento
}

\section{Introduction}

1 Une tâche essentielle des enseignants est de rechercher, de fabriquer, de sélectionner, voire de modifier des ressources qu'ils présentent à leurs apprenants mais aussi qu'ils partagent avec leurs collègues. Dans cette perspective, la question de la place du manuel comme outil utilisé par les enseignants de langues se pose. Plusieurs contraintes régissent l'élaboration d'un manuel : les textes officiels, les progrès des connaissances, les modes en pédagogie et didactique et les besoins de la société (Lebrun, dir. 2006; VerdelhanBourgade \& Auger, 2011). Pour certains, le manuel est donc une illustration du processus de transposition didactique, un intermédiaire entre ce qui est prescrit, les savoirs savants et les pratiques professionnelles (Perrenoud, 1998; Bruillard, 2005). Il constitue aussi parfois un outil de formation des enseignants et même pour certains le seul élément de formation continue.

Dans cet article, je propose de montrer les différents facteurs qui influent sur les pratiques liées à l'utilisation des manuels par les professeurs de FLE (Français Langue Étrangère. J'interrogerai plus particulièrement les attitudes et les comportements des enseignants vis-à-vis des manuels.

\section{Corpus et méthodologie}

3 Le corpus est constitué de 16 entretiens semi-directifs avec des enseignants de FLE : 9 enseignants novices (moins de 5 ans d'expérience) et 7 enseignants experts (plus de 5 ans d'expérience). Le questionnaire d'enquête (cf. annexe en fin d'article) était composé d'une 
série de questions sur les caractéristiques personnelles et professionnelles des enseignants, leurs pratiques, leurs opinions, leurs représentations sur les manuels et leurs usages. Il s'agissait de laisser libre cours aux choix de réponse des personnes interrogées, avec leurs mots et des détails faisant sens selon eux. Cette méthode permet le questionnement sur la complexité des objets étudiés. Pour cela, j’ai évité les questions trop précises en dialoguant sur le mode de la conversation et en relançant sans orienter.

Les enseignants interrogés ont pour la plupart fait leurs études à l'université Paris Descartes (master de didactique du français langue étrangère et interculturalité) ou à l'université Sorbonne Nouvelle - Paris 3 (master de didactique du français et des langues) et travaillent aujourd'hui dans différentes institutions à Paris (Alliance Française Paris Île de France, mairie de Paris, universités, associations) ou à l'étranger (écoles de langue, universités). Si tous ces enseignants ont aujourd'hui une formation en FLE, certains ont commencé à enseigner avant d'avoir suivi un cursus universitaire. Ils ont tous essentiellement enseigné à des adultes. Le tableau ci-dessous résume les différentes données personnelles et professionnelles des personnes interrogées tout en préservant leur anonymat.

Tableau 1 - Informations personnelles et professionnelles des enseignants interrogés

\begin{tabular}{|c|c|c|c|c|c|}
\hline Enseignants & Âge & Études et lieu & $\begin{array}{l}\text { Années } \\
\text { d'expérience }\end{array}$ & $\begin{array}{l}\text { Novices/ } \\
\text { experts }\end{array}$ & Lieux d'exercices \\
\hline A & 32 & $\begin{array}{lrr}\text { Master } & 2 & \text { FLE } \\
\text { université } & \text { Paris } \\
\text { Descartes } & & \end{array}$ & $\begin{array}{l}2 \quad \text { ans sans } \\
\text { formation }\end{array}$ & Novice & $\begin{array}{l}\text { Écoles de langue, } \\
\text { entreprise }\end{array}$ \\
\hline B & 57 & $\begin{array}{lrr}\text { Master } & 2 & \text { FLE } \\
\text { université } & \text { Paris } \\
\text { Descartes } & & \end{array}$ & $\begin{array}{l}16 \text { ans, dont } 8 \text { sans } \\
\text { formation }\end{array}$ & Expert & $\begin{array}{l}\text { Écoles de langue, } \\
\text { Université }\end{array}$ \\
\hline C & 43 & $\begin{array}{lrr}\text { Master } & 2 & \text { FLE } \\
\text { université } & \text { Paris } \\
\text { Descartes } & & \end{array}$ & 6 mois & Novice & $\begin{array}{l}\text { École de langue, } \\
\text { association }\end{array}$ \\
\hline D & 50 & $\begin{array}{lr}\text { Maîtrise } & \text { FLE } \\
\text { université } & \text { Paris } \\
\text { Descartes } & \end{array}$ & $\begin{array}{l}9 \text { ans dont } 1 \text { an sans } \\
\text { formation }\end{array}$ & Expert & $\begin{array}{l}\text { Universités, } \\
\text { association }\end{array}$ \\
\hline$E$ & 26 & $\begin{array}{l}\text { Master } 2 \text { université } \\
\text { Paris Descartes }\end{array}$ & 5 ans & Expert & $\begin{array}{l}\text { Universités, } \\
\text { associations, école } \\
\text { de langue }\end{array}$ \\
\hline $\mathrm{F}$ & 34 & $\begin{array}{l}\text { Master } 2 \text { université } \\
\text { Paris Descartes }\end{array}$ & 5 ans & Expert & $\begin{array}{l}\text { École de langue, } \\
\text { mairie, université }\end{array}$ \\
\hline G & 39 & $\begin{array}{l}\text { Maitrise FLE et } \\
\text { master en ingénierie } \\
\text { FLE U } \\
\text { université Sorbonne } \\
\text { Nouvelle }\end{array}$ & 13 ans & Expert & $\begin{array}{l}\text { Universités, mairie, } \\
\text { écoles de langue }\end{array}$ \\
\hline
\end{tabular}




\begin{tabular}{|c|c|c|c|c|c|}
\hline $\mathrm{H}$ & 31 & $\begin{array}{l}\text { Cesop, Dpafp, Daefle }{ }^{1} \\
\text {, Master } 2 \text { université } \\
\text { Paris Descartes }\end{array}$ & 3 ans & Novice & École de langue \\
\hline I & 27 & $\begin{array}{l}\text { Master } 2 \text { université } \\
\text { Paris Descartes }\end{array}$ & 4 ans & Novice & $\begin{array}{l}\text { Universités, écoles } \\
\text { de langue }\end{array}$ \\
\hline $\mathrm{J}$ & 56 & $\begin{array}{l}\text { Master } 2 \text { université } \\
\text { Paris Descartes }\end{array}$ & 9 ans & Expert & $\begin{array}{l}\text { École de langue, } \\
\text { Mairie, universités }\end{array}$ \\
\hline K & 33 & $\begin{array}{l}\text { Master } 2 \text { université } \\
\text { Paris Descartes }\end{array}$ & $\begin{array}{l}4 \quad \text { ans sans } \\
\text { formation et } 1 \text { an } \\
\text { avec formation }\end{array}$ & Expert & $\begin{array}{l}\text { Cours particulier, } \\
\text { écoles de langue, } \\
\text { associations }\end{array}$ \\
\hline $\mathrm{L}$ & 25 & $\begin{array}{l}\text { Master } 2 \text { université } \\
\text { Sorbonne Nouvelle }\end{array}$ & 9 mois & Novice & Association \\
\hline M & 28 & $\begin{array}{l}\text { Master } 2 \text { université } \\
\text { Paris Descartes }\end{array}$ & 1 an & Novice & École de langue \\
\hline $\mathrm{N}$ & 32 & $\begin{array}{llr}\text { Master } & 2 & \text { FLE } \\
\text { université } & \text { Sorbonne } \\
\text { Nouvelle } & \end{array}$ & 2 ans & Novice & Écoles de langue \\
\hline 0 & 35 & $\begin{array}{l}\text { Master } 2 \text { université } \\
\text { Paris Descartes }\end{array}$ & 2 ans & Novice & FLE en entreprise \\
\hline$P$ & 24 & $\begin{array}{llr}\text { Master } & 2 & \text { FLE } \\
\text { université } & \text { Sorbonne } \\
\text { Nouvelle } & & \end{array}$ & 18 mois & Novice & $\begin{array}{l}\text { Université, } \\
\text { association }\end{array}$ \\
\hline
\end{tabular}

5 Cette recherche se situe à la fois dans la lignée des travaux de Rabardel (1995) et de Rabardel \& Pastré dir. (2005) sur les genèses instrumentales et des travaux de Leplat (1992, 1997), Goigoux (1997) sur des concepts et des méthodes de la psychologie ergonomique, ces choix permettent de garder l'inscription professionnelle de l'activité des enseignants. Dans cette perspective, les manuels constituent des ressources qui ont une influence sur leurs tâches au moment de la planification de l'action mais aussi au moment de la réalisation de l'action (Goigoux, 2007b). À la suite de Rabardel (1995), j'examine les manuels en fonction de l'utilisation qu'en font les enseignants. Pour Goigoux (2007a: 60), ces manières d'utiliser les manuels résultent d'une construction personnelle et de l'appropriation de schèmes professionnels préexistants. Ils ont une dimension privée puisqu'ils sont propres à chaque individu et s'inscrivent dans la mémoire personnelle des sujets en tant que ressources mobilisables. Mais ils ont également une dimension sociale : communs aux membres d'un même milieu de travail, il convient de les considérer comme des schèmes sociaux d'utilisation, c'est-à-dire des ressources inscrites dans la mémoire impersonnelle du collectif professionnel. À ce titre, ils contribuent à définir le genre professionnel. Ainsi l'usage du manuel se trouve au cœur de plusieurs tensions: les exigences normatives du manuel, les prescriptions professionnelles et les efforts que fait l'enseignant pour adapter le contenu des manuels (Rabardel, 1995 : 14). 
6 La méthode d'analyse de contenu des données recueillies a consisté en une analyse thématique (Bardin, 2007). Pour le traitement des données, il s'est agi de repérer les éléments significatifs du discours des enseignants puis de les catégoriser. Différents axes ont été dégagés : le choix des ressources en fonction des acteurs, le choix des ressources en lien avec la planification et enfin le choix des ressources en fonction des finalités professionnelles, sociales mais aussi personnelles.

\title{
Manuels et acteurs de la formation
}

7 À l'instar de Leplat (1992) et Goigoux (2007a), je dirais que l'activité de l'enseignant, en ce qui concerne les manuels, prend trois directions: vers les apprenants, vers les autres acteurs de la scène d'enseignement/apprentissage et vers lui-même.

\section{L'agir enseignant dirigé vers les apprenants}

8 Le professeur vise à faciliter les apprentissages des apprenants tant au niveau cognitif que social. Tous les enseignants que j'ai interrogés indiquent choisir leurs manuels en fonction du public: âge, niveau de langue, nationalité et adapter ou sélectionner les activités en fonction de ces critères.

\begin{abstract}
Moi j'ai vraiment tendance à toujours utiliser les mêmes types de documents mais après la séquence pédagogique elle est pas figée parce que même si je prends deux publics FLE, si je prends par exemple, l'exemple de Paris 5 et l'exemple de sciences po, c'est des publics FLE mais nous on va pas avoir la même approche parce que à sciences po ils sont en master et à Paris 5 voilà ils sortent du bac (extrait de l'entretien avec l'enseignant $G$ ).

J'utilise mon manuel de manière différente en fonction du public. Forcément si on a une classe où les étudiants sont plus âgés, le rythme ne va pas être identique qu'avec des étudiants plus jeunes mais je vais aussi changer les activités. Et puis, il y a des classes qu'on a besoin de pousser plus (extrait d'entretien avec l'enseignante P).
\end{abstract}

9 L'enseignant peut aussi être amené à prendre en compte les désirs des apprenants. Par exemple, plusieurs enseignants interrogés $(\mathrm{B}, \mathrm{D}, \mathrm{E}, \mathrm{J})$ ont fait remarquer que s'ils n'aiment pas trop s'attacher à un manuel, ce n'est pas le cas des apprenants qui préfèrent en avoir un et le suivre pas à pas. Certains enseignants tentent de répondre partiellement à cette demande surtout lorsqu'elle est renforcée par l'institution.

[...] Malgré tout je pense que les gens qui veulent commencer avec une méthode, ils ont cette envie de lire cette méthode, ils l'ont ce livre, ils l'ont chez eux, ils ont envie de voir qu'ils ont lu toutes les pages, qu'on n'a pas laissé des images. Alors on l'utilise mais ils comprennent pas si on laisse un document à part. J'essaie toujours d'expliquer pourquoi j'utilise ou pourquoi j'utilise pas voilà pourquoi j'amène un autre document. En quoi je trouve que c'est pertinent de faire cet exercice ou de ne pas le faire. J'essaie toujours d'expliquer malgré tout parce que c'est ce qui leur manque c'est l'explication (extrait d'entretien avec l'enseignante E). 


\section{L'agir enseignant dirigé vers les autres acteurs de la scène d'enseignement/ apprentissage} soit acceptable pour sa hiérarchie, ses collègues qui interviennent auprès des apprenants visés mais aussi les autres enseignants qui agissent dans le même lieu de travail (école de langue, mairie...). Dans le corpus, plusieurs enseignants interrogés indiquent alimenter les manuels qu'ils suivent en injectant de nouvelles activités $(\mathrm{E}, \mathrm{H}, \mathrm{I}, \mathrm{J})$ tout en les suivant relativement pas à pas parce qu'ils sont plusieurs à travailler sur le même manuel avec les apprenants.

\footnotetext{
Les manuels FLE, on se partage les unités avec les enseignants, on partage pas les pages. Et on fait tous les points langagiers et on utilise une bonne partie des supports mais pas forcément tous parce que dans les manuels on a la conceptualisation à côté qui est toute prête ou bien on a les exercices avec les réponses à la fin, on a les transcriptions des compréhensions orales qui peuvent être vues à l'avance par les étudiants donc j'aime mieux apporter des éléments autour du thème ou les transformer (extrait d'entretien avec l'enseignante J).
}

D'autres professeurs (A, D, N) indiquent suivre les directives de la hiérarchie même s'ils ne sont pas d'accord.

Je n'étais pas forcément d'accord avec ce qu'on m'imposait mais après faut s'adapter (extrait d'entretien avec l'enseignante D).

Enfin, deux enseignants refusent les manuels imposés, comme l'enseignante B qui indique que

le seul endroit où on m'a imposé quelque chose c'était à l'Alliance Française.

J'ai dit oui mais je l'ai pas fait.

et l'enseignant $G$ qui déclare avoir refusé de travailler dans une institution parce que justement elle imposait un manuel. Il a fait le choix d'intégrer des établissements qui le laissaient libre des ressources et des contenus utilisés.

\section{L'agir enseignant dirigé vers lui-même}

Plusieurs enseignants (F, I, K, M, P) font des choix de manuels, d'activités dans les manuels ou non en fonction de la satisfaction qu'ils vont en retirer en termes d'objectifs pédagogiques, de valeurs, de fierté professionnelle, d'intégration à leur milieu de travail, de reconnaissance sociale. Par exemple, I indique n'utiliser que des documents authentiques qu'il aime, $\mathrm{K}$, quant à elle, déclare avoir une auteure préférée (Claire Miquel) parce qu'elle trouve que ses dialogues sont très bien écrits. $\mathrm{F}$ et $\mathrm{P}$ transforment beaucoup les documents qu'elles utilisent sinon elles ont l'impression qu'elles n'ont pas travaillé et que le cours n'a pas été préparé. On voit ainsi des choix se mettre en place selon leurs propres intérêts mais ces intérêts sont aussi en lien avec ce qu'ils estiment être leurs devoirs d'enseignants. 


\section{Manuels et planification} progressions proposées dans les manuels, des tableaux des contenus, des tables des matières, notamment lorsque qu'ils n'ont pas le Cecrl (Cadre Européen Commun de Référence pour les Langues, Conseil de l'Europe, 2001) à portée de main ou qu'ils le trouvent trop compliqué (B, C, F, J, K, N, P). Les propos de l'enseignante $\mathrm{C}$ résument bien cette pratique :

Je trouve que tout ce qui est table des matières c'est très très très utile, ne serait-ce que ça [...] parce qu'on peut pas avoir toujours sous le nez le Cecr et puis c'est pas toujours hyper précis sur comment faire les choses. Je trouve que ne serait-ce que regarder les tables des matières puis voir si telle chose est introduite au même moment. Ça peut être bien pour se rassurer. [...] Voilà, je trouve que les tables des matières aident à la progression.

La plupart indiquent aménager/adapter les activités (A, B, C, F, G, I, J), ou changer les consignes, les questions de compréhension... (A, C, I, J). Ils piochent aussi dans différentes méthodes ce qui les intéresse (documents, activités, illustrations), mais certains disent aussi s'en servir relativement peu et chercher constamment des documents authentiques (chansons, poésies, textes littéraires, documents sur internet...) pour construire leurs cours (B, C, G, I, J). Les paroles de l'enseignante $\mathrm{C}$ illustrent ces manières de faire :

Même si je suis en accord avec la méthodologie du manuel, je ne vais pas le suivre pas à pas [...], je vais piocher dans d'autres manuels, il peut y avoir des illustrations, des aide-mémoire qui étaient pas mal [...] Je me servais des exercices parfois tels quels et parfois je les remaniais pour qu'ils soient contextualisés, voilà [...] et sinon je me sers beaucoup des sites internet.

Le témoignage de l'enseignante $\mathrm{E}$ atteste des transformations que les enseignants sont amenés à faire lorsqu'ils construisent leurs cours afin de les adapter au public.

Il faut être capable de déterminer les points qu'on va travailler, tout ce qu'il faut et essayer de composer avec ça, c'est du bricolage, du découpage, du collage. Voilà, j'ai une espèce d'étendue de bureau sinon je n'y arrive pas.

Différentes recherches montrent combien les connaissances disciplinaires des enseignants jouent un rôle essentiel dans leurs activités de préparation des cours (Maury, 1992 ; Vantourout \& Maury, 2006). Par exemple, dans le corpus, l'enseignant P indique se sentir plus sûr de lui dans le domaine du vocabulaire que dans le domaine grammatical, et donner facilement libre cours à son imagination pour concevoir des situations permettant d'enrichir le vocabulaire, alors qu'en grammaire, il choisira de proposer seulement les activités du manuel pour lesquelles il a la correction dans le guide pédagogique.

La planification d'un cours à partir d'un manuel est aussi liée à l'expérience des enseignants : lorsqu'ils débutent ou qu'ils n'ont pas d'expérience, ils indiquent utiliser de manière relativement linéaire le manuel en le suivant pas à pas (A, E, K) voire à se fier au guide pédagogique. L'enseignante $\mathrm{E}$ explique à propos des manuels :

Je me rends compte maintenant qu'au début je les suivais pas à pas, ça c'est sûr. Je faisais beaucoup plus confiance au livre en lui-même parce que j'avais moins d'expérience et aussi parce que je pensais qu'il était parfait. 
L'enseignante A va dans le même sens lorsqu'elle dit :

J'ai commencé par commencer à la première page pour terminer à la dernière. J'ai jamais sauté de leçon et je suis jamais revenu en arrière parce qu'il me semblait que la progression était logique. Aujourd'hui, avec l'expérience et puis la formation qu'on a reçue ici j'aurais tendance à sélectionner ce qui m'intéresse et à laisser ce qui ne m'intéresse pas.

20 On voit ainsi que la gestion de l'utilisation du manuel pour la planification varie en fonction de différents paramètres qui peuvent se croiser comme l'ancienneté dans l'enseignement ou encore les savoirs acquis dans les domaines convoqués.

\section{Manuels et finalités professionnelles, sociales et personnelles}

21 Les pratiques d'un enseignant apparaissent comme un ensemble organisé d'activités qui s'exercent en un lieu précis et à un moment donné, en référence à un contexte institutionnel et social, et avec une inscription dans l'histoire personnelle de l'enseignant. Ces activités visent la réalisation d'objectifs qui correspondent à différentes finalités professionnelles et personnelles. Comme le suggèrent Bru (2002) puis Roditi (2011), cela conduit à ne pas envisager les pratiques enseignantes comme déterminées par différents facteurs, au sens où, par exemple, elles seraient l'effet de règles, de choix, de décisions, etc., il semble donc plus adéquat d'envisager la pratique d'un enseignant comme étant organisée par des processus plus globaux, à un moment donné, pour une situation rencontrée dans des contextes institutionnels et sociaux particuliers, et qui s'ajuste éventuellement dans l'interaction avec les apprenants ou d'autres acteurs du système. Par exemple, tous les enseignants indiquent adapter les activités dans les manuels en fonction des institutions mais surtout en fonction des apprenants qu'ils ont en face d'eux : selon leur nationalité, leur niveau, leurs attentes, leurs objectifs...

Dans une approche didactique et ergonomique, l'organisation des pratiques peut donc être considérée selon trois dimensions qu'on peut qualifier d'organisatrices des pratiques enseignantes (Robert \& Rogalski, 2002; Roditi, 2011) : les dimensions institutionnelle, sociale et personnelle.

\section{Dimension institutionnelle}

23 Au niveau de la dimension institutionnelle des pratiques enseignantes, les enquêtes montrent que beaucoup d'institutions dans lesquelles ont travaillé les enseignants interrogés n'imposent pas de méthodologie particulière et laissent les enseignants relativement libres du matériel utilisé et aussi des méthodologies.

Cependant, pour les institutions qui en imposent, lorsque les enseignants n'ont pas ou peu de formation avant d'enseigner $(\mathrm{A}, \mathrm{E}, \mathrm{F}, \mathrm{H})$, ils ont tendance à se plier à ce que demande l'institution au niveau des méthodologies et des programmes. Par exemple l'enseignante A a adopté la méthode directe imposée par l'école de langue dans laquelle elle travaillait. Les enseignants plus chevronnés refusent souvent cette obligation et organisent leurs cours comme ils l'entendent (B) voire partent des institutions qui imposent méthodologies et méthodes $(B, G)$. 

méthodologie (C, H, I).

De fait, avec un manuel, on nous impose une méthodologie en français général parce qu'Alter Ego le premier, c'était une méthode communicative et puis Alter Ego + que j'ai expérimenté restait communicatif mais il y avait quand même des propositions de projets (extrait d'entretien avec l'enseignante $\mathrm{H}$ ).

Ils sont aussi conscients des contradictions dans certaines institutions comme celles qui imposent une méthodologie mais avec un programme qui ne correspond pas à cette méthodologie. Par exemple, l'enseignante D indique que l'institution dans laquelle elle travaillait en Angleterre imposait une approche communicative mais l'aspect grammatical était le seul point pour valider la maîtrise de la langue ou cette autre institution où travaille l'enseignante $\mathrm{E}$ et qui l'oblige à utiliser un manuel dont le contenu n'est pas en lien avec la méthodologie imposée.

Pour les enseignants, les situations professionnelles dans lesquelles ils sont engagés correspondent en partie à des obligations. Dans les systèmes d'enseignement auxquels appartiennent les professeurs que j'ai questionnés, ces obligations figurent dans le Cecrl, elles sont aussi issues de leur formation universitaire et sont constituées des orientations données par l'institution. Même si, pour les enseignants, ces obligations ne sont pas toujours précises, notamment à cause des différences de contexte, on peut les interpréter comme des tâches prescrites qu'ils redéfinissent personnellement ou collectivement. Les enseignantes $\mathrm{A}$ et $\mathrm{E}$ ayant travaillé dans des écoles de langue pratiquant la méthode directe $^{2}$ ont signalé que ces institutions imposaient des manuels créés par les institutions elles-mêmes. Ces manuels, en accord avec la méthode imposée, ne présentaient pas d'activités grammaticales. Afin d'être en accord avec leurs propres conceptions de l'enseignement des langues, les deux enseignantes en question ont décidé de suivre les manuels tels qu'ils étaient imposés mais en ajoutant des activités grammaticales issues d'autres manuels ou de matériel complémentaire. Elles ont toutes deux indiqué qu'un bon enseignement de la langue doit passer par un apprentissage grammatical tout en précisant que bien souvent la demande d'un enseignement de la grammaire venait aussi des apprenants.

\begin{abstract}
J'utilise d'autres méthodes, j'utilise différentes grammaires, j'utilise d'autres documents, je me sers des cours que j'ai faits avant, de ce qu'on a appris et j'essaie de créer complètement mes propres cours sur la base des leçons de la méthode, c'est-à-dire que je suis malgré tout la progression parce que j'ai signé pour travailler chez eux et c'est comme ça qu'ils fonctionnent mais j'essaie malgré tout de créer un cours intéressant et pour lequel en tout cas j'ai l'impression d'apporter quelque chose. [...] Il faut savoir que les Allemands ont vraiment besoin de la règle [grammaticale]. Ils veulent faire de la grammaire (extrait d'entretien avec l'enseignante E).
\end{abstract}

La dimension institutionnelle pour les professeurs de FLE ne se réfère pas seulement à l'institution dans laquelle ils travaillent, mais aussi à l'institution productrice des savoirs. Ces professeurs ont en effet été formés à l'université par des enseignants-chercheurs. Les enseignants possèdent un rapport de spécialiste à leur discipline qui les engage dans leur enseignement vis-à-vis de cette institution productrice des savoirs ce qui explique la méfiance d'une partie des enquêtés envers les manuels, une méfiance née des études où les universitaires sont très critiques et apprennent aux futurs enseignants à être eux- 
mêmes très critiques envers les manuels $(G, K)$. L'enseignant $G$ remarque que le master FLE donne une vision globale de ce que doit être le métier d'enseignant. Il y a une prise de conscience de l'importance des quatre compétences (compréhension orale, compréhension écrite, expression orale et production écrite) avec une place limitée de la grammaire. Il remarque la distance qui existe entre les collègues formés à l'université et ceux qui n'ont pas de formation. La langue n'est pas abordée de la même manière et il donne comme exemple l'enseignement de la grammaire qui devrait, selon lui, privilégier une approche contextualisée, inductive et explicite et non pas partir d'un point qui sera développé in extenso comme cela est appliqué par les enseignants sans formation.

Il arrive que ces deux composantes de la dimension institutionnelle - l'institution dans laquelle travaillent les enseignants et l'institution productrice des savoirs - entrent en concurrence et que cela engendre un problème professionnel pour certains d'entre eux. Par exemple, les deux enseignantes citées plus haut, A et E, qui indiquent que leur institution impose une méthode directe en opposition avec ce qu'elles ont appris à l'université, on voit naitre ainsi une tension qui les conduit généralement à arranger des séquences pour échapper à cette approche, en proposant, par exemple, des activités de conceptualisation grammaticale.

\section{Dimension sociale}

30 Au niveau de la dimension sociale des pratiques enseignantes, comme cela a déjà été mentionné, les tâches que les enseignants réalisent ne correspondent pas toutes à des obligations professionnelles à proprement parler. Ils s'en imposent certaines de manière collective en fonction de normes admises qui peuvent varier selon les contextes dans lesquels ils travaillent et les réseaux relationnels auxquels ils appartiennent. Cela confère une dimension sociale à leur pratique. Ainsi, les contextes considérés pour comprendre l'activité de l'enseignant ne se réduisent pas à la classe, à son niveau, à son effectif et à sa composition plus ou moins hétérogène. Parce qu'il exerce dans une institution, l'enseignant dépend aussi de ses collègues. Par exemple, les enseignants A, G et N, peu enclins à utiliser des manuels, vont tout de même le faire parce que les autres collègues décident de monter des programmes associés aux manuels ou encore parce qu'au contact des collègues qui utilisent différents manuels, leurs représentations sur ces mêmes manuels vont changer.

Ce sont mes collègues qui m'ont appris à savoir dompter les manuels sans pour autant les utiliser parce que ça me faisait un petit peu peur, je disais mais moi je sais enseigner mais je sais pas enseigner avec un manuel. Je l'ai souvent dit. Aujourd'hui, ça je le dis plus parce que je me rends compte que ça veut rien dire enseigner avec un manuel (extrait d'entretien avec l'enseignant $\mathrm{G}$ ).

C'est vrai que je ne suis pas trop pour utiliser les manuels mais mes collègues préfèrent alors quand je monte des leçons avec elles, je les utilise un peu (extrait d'entretien avec l'enseignante $\mathrm{N}$ ).

31 L'enseignant appartient bien à un milieu professionnel plus vaste, où on trouve des manières d'agir collectivement construites. En même temps, ces manières d'agir facilitent l'exercice du métier car il n'a pas à tout inventer, son activité repose sur des habitudes de la profession, et cela le rend davantage disponible pour s'adapter aux situations 
particulières qu'il rencontre. Elles contraignent aussi son action qui est nécessairement référée au métier et qui, d'une certaine manière, engage toute la profession quand il agit. Plusieurs enseignants font remarquer que l'usage du manuel guidé par un coordonnateur ou par un collègue leur a grandement facilité la tâche soit parce qu'ils ont commencé à enseigner sans formation et que cela les rassurait, soit parce qu'ils étaient débutants et qu'ils avaient besoin de ce guidage (A, $\mathrm{H}, \mathrm{K}$ et $\mathrm{O})$.

C'est vrai que quand j'ai commencé, c'était rassurant d'avoir la responsable qui me disait quoi faire avec les manuels. J'en avais vraiment besoin parce que j'étais complétement perdue (extrait d'entretien avec l'enseignante 0 ).

\section{Dimension personnelle}

La troisième dimension organisatrice des pratiques enseignantes est donc liée à l'enseignant lui-même. Des informations, comme les appartenances culturelles et sociales de l'enseignant, la manière dont il a été "enseigné" lui-même, permettent de comprendre ses pratiques (Roditi, 2011 : 102-106). Ces contraintes liées à la dimension personnelle vont agir comme un "filtre d'habitus et de croyance" (Perrin-Glorian, 1995) qui va influer sur les pratiques autour du manuel. Par exemple, certains enseignants (B, E, I, K et O) indiquent choisir dans les manuels les documents en lien avec l'intérêt des apprenants mais aussi les leurs. Il n'est pas question de choisir un document, un film ou une chanson qu'ils n'aiment pas ou qui va à l'encontre de leur philosophie. D'autres (C, H, I, K et M) soulignent qu'ils ont pris pour modèles des professeurs qu'ils ont eus lors de leurs apprentissages divers (en langues bien sûr, mais aussi en lettres ou encore en primaire) et qu'ils essaient de reproduire leurs manières d'être et de faire, tout en tentant de rester en accord avec les méthodologies pratiquées, par exemple l'enseignante $\mathrm{H}$ explique son expérience d'apprentissage de l'anglais et l'impact que cela a eu sur sa manière d'enseigner ensuite :

J'ai eu un très bon prof d'anglais et qui lui nous faisait faire des jeux de rôle à chaque cours. Il nous levait en début de cours. C'était quelqu'un d'hyper rigide mais très fort dans son apprentissage [...]. Il m'a influencée mais pas dans la manière d'être parce qu'il était ultra rigide mais j'ai appris que c'était important d'apprendre une langue, d'apprendre à parler une langue en même temps qu'on apprenait à l'écrire.

\section{Conclusion}

En français langue étrangère, une tâche centrale des enseignants est de chercher et de sélectionner les ressources qu'ils présentent à leurs apprenants et qui servent de support à leurs activités. Nous avons très peu de données sur cette activité caractéristique de leur liberté pédagogique. L'analyse de l'usage que ces enseignants font des manuels constitue un enjeu pour la compréhension du système qu'ils mettent en place et de ses évolutions. L'objectif de cette recherche était de pallier le manque d'informations sur la gestion des manuels par les enseignants. Ainsi, en conclusion, on peut dire que plusieurs composantes entrent en jeu dans l'usage des manuels par les enseignants (Robert \& Rogalski, 2002): les composantes personnelle (représentations, conceptions des enseignants sur le FLE, sur l'enseignement/apprentissage et sur le public), 
institutionnelle, cognitive (nature et organisation des contenus) et sociale (contraintes liées à la classe, aux collègues, aux programmes et à l'institution). Toutes ces composantes interviennent lorsque l'enseignant prépare son cours à partir de manuels. Les quelques exemples cités montrent aussi que ces trois dimensions s'entrecroisent, interagissent et conduisent ces enseignants à mettre en œuvre des savoirs construits mais aussi à bricoler soit pour adhérer à leurs propres représentations de l'enseignement des langues soit pour répondre à la demande des institutions, des collègues ou encore des apprenants. La prise en compte des différentes dimensions envisagées permet de mieux appréhender les pratiques autour de la gestion des manuels. Les résultats de cette recherche indiquent que l'utilisation des manuels peut modifier l'expérience et éventuellement les représentations des enseignants et donc par extension avoir une influence sur leurs pratiques mais pas de manière aussi systématique qu'on aurait pu penser au premier abord. L'enseignant a des représentations, des finalités mais aussi une expérience professionnelle qui le conduit à repenser, organiser et transformer les activités proposées dans les manuels ou tout simplement à ne pas les utiliser.

\section{BIBLIOGRAPHIE}

Bardin, L. (2007). L'analyse de contenu. Paris : Presses Universitaires de France.

Bru, M. (2002). "Pratiques enseignantes : des recherches à conforter et à développer". Revue française de pédagogie, $\mathrm{n}^{\circ}$ 138. pp. 63-73.

Bruillard, E. (2005). "Les manuels scolaires questionnés par la recherche". In Bruillard, E. (dir.). Manuels scolaires, regards croisés. Caen : Crdp de Basse-Normandie. pp. 13-36.

Conseil de l'Europe (2001). Cadre européen commun de référence pour les langues. Paris : Didier.

Goigoux, R. (1997). "La psychologie cognitive ergonomique : un cadre d'étude des compétences professionnelles des enseignants de Français". In Bautier, E. \& Bucheton, D. (dir.). Pratiques enseignantes et activités des élèves dans la classe de Français, Didactique du Français Langue Maternelle (Dflm), $\mathrm{n}^{\circ}$ 21. pp. 56-61.

Goigoux, R. (2007a). "Un modèle d'analyse de l'activité des enseignants". Éducation et didactique, vol. 1, $\mathrm{n}^{\circ}$ 3. pp. 47-69. Disponible en ligne. http://educationdidactique.revues.org/232?lang=en consulté le 25/11/2012.

Goigoux, R. (2007b). "Rien de plus pratique qu'une bonne théorie? Si, deux ! Les concepts de schème et de genre au service d'une analyse didactique de l'activité d'enseignement". In Merri, M. (dir.). Activité humaine et conceptualisation : questions à Gérard Vergnaud. Toulouse : Presses Universitaires du Mirail. pp. 91-102.

Lebrun, M. (dir.). (2006). Le manuel scolaire, un outil à multiples facettes. Québec : Presses de l'Université du Québec.

Leplat, J. (1992). L'analyse du travail en psychologie ergonomique (2 tomes). Toulouse : Octarès.

Leplat, J. (1997). Regards sur l'activité en situation de travail. Paris : Presses Universitaires de France. 
Maury, S. (1992). "La représentation du savoir chez l'enseignant, source de difficultés dans l'enseignement de certaines connaissances ?". Tréma, $\mathrm{n}^{\circ}$ 1. pp. 13-75.

Perrenoud, P. (1998). "La transposition didactique à partir de pratiques : des savoirs aux compétences". Revue des sciences de l'éducation, vol. XXIV, n³ 3. pp. 487-514.

Perrin-Glorian, M.-J. (1995). Actes de l'école d'été. Actes de la VIIIe École d'Été de didactique des mathématiques. Clermont-Ferrand : Irem de Clermont-Ferrand.

Rabardel, P. (1995). Les hommes et les technologies. Approche Cognitive des instruments contemporains. Paris : Armand Colin.

Rabardel, P. \& Pastré, P. (dir.). (2005). Modèles du sujet pour la conception. Dialectiques activités développement. Toulouse : Octarès.

Robert, A. \& Rogalski, J. (2002). "Le système complexe et cohérent des pratiques des enseignants de mathématiques : une double approche". Revue canadienne de l'enseignement des sciences, des mathématiques et des technologies, vol. 2, $n^{\circ}$ 4. pp. 505-528.

Roditi, E. (2011). Recherches sur les pratiques enseignantes en mathématiques : apports d'une intégration de diverses approches et perspectives. Synthèse de HDR. Université Paris Descartes.

Vantourout, M. \& Maury, S. (2006). "Quelques résultats relatifs aux connaissances disciplinaires de professeur stagiaires dans des situations d'évaluation formative". Revue des sciences de l'éducation, vol. 32, $\mathrm{n}^{\circ}$ 3. pp. 759-782.

Verdelhan-Bourgade, M. \& Auger, N. (2011). "Les manuels et supports pédagogiques : catégorisations". In Blanchet, P. \& Chardenet, P. (dir.). Guide pour la recherche en didactique des langues et des cultures. Approches contextualisées. Paris : Archives contemporaines. pp. 307-312.

\section{ANNEXES}

\section{Questionnaire d'enquête auprès des enseignants de FLE}

Nom de l'enseignant :

Âge :

Formation :

En même temps ou après formation?

Nombre d'années de pratique dans le FLE ou ailleurs dans l'enseignement :

Quel type de public?

Nom des institutions, organismes, associations (FLE/FLS $3 / \mathrm{FLM}^{4}$ ) et temps d'expérience dans chacune d'elle :

Y-a-t-il une approche méthodologique imposée par l'institution actuelle ? Si oui laquelle?

L'enseignant est-il en accord avec cette approche ? En quoi ? Pourquoi ?

Quels manuels de FLE sont utilisés dans la pratique?

Y-a-t-il un manuel imposé (ou des) par l'organisme?

L'enseignant a-t-il un manuel préféré (ou des) ? Si oui lequel ? Pourquoi ? 
Comment sont utilisés les manuels : In extenso ? Ponctuellement ? Plusieurs à la fois ? En lien avec le guide pédagogique ? Pas à pas ? Réaménagé ? Exemple.

La pratique de l'enseignant a-t-elle évolué ou évolue-t-elle en fonction du contenu des manuels? Si oui, au niveau des contenus? Au niveau de la méthodologie? Comment? Exemples.

Est-ce que l'enseignant a différentes manières d'utiliser un même manuel en fonction de publics différents?

Les manuels utilisés par l'enseignant sont-ils proches de l'approche imposée par l'institution? Explication.

Est-ce que l'enseignant utilise des méthodes complémentaires, lesquelles?

Discussion autour d'une séquence de grammaire préparée à partir d'un manuel (demande faite au préalable).

Question autour des démarches d'enseignement/d'apprentissage : inductif/déductif/ explicite/ implicite

Différentes manières d'enseigner en fonction du public (didactique, attitude/ comportement, contenu...).

Qu'est-ce qui a le plus influencé l'enseignant dans sa manière d'enseigner (expérience vécue en collège/lycée, formation, stages...) ?

\section{NOTES}

1. Cesop : CErtificat de Stage d'Observation et de Pratique en français langue étrangère.

Dpafp: Diplôme Professionnel de l'Alliance Française Paris Ile-de-France en français langue étrangère.

Daefle : Diplôme d'Aptitude à l'Enseignement du Français Langue Étrangère.

2. La méthode directe consiste notamment à enseigner la langue sans passage par la traduction et sans enseignement direct de la grammaire.

3. Français langue seconde.

4. Français langue maternelle.

\section{RÉSUMÉS}

À partir de l'analyse d'entretiens menés auprès d'enseignants de FLE (Français Langue Étrangère) débutants et chevronnés sur l'utilisation des manuels, nous verrons quels facteurs institutionnels (contraintes liées aux lieux de travail et aux institutions productrices de savoirs), sociaux (contraintes liées à la classe, aux collègues, aux programmes et à l'institution) et personnels 
(représentations sur le FLE, sur l'enseignement/apprentissage et sur les apprenants) influent sur les pratiques liées à cette utilisation.

This paper reports on an analysis of interviews carried out with novice and experienced teachers of French as a foreign language about their use of text books. We will seek to identify institutional factors (constraints related to work places, programs and knowledge-producing institutions), social factors (constraints related to learners, colleagues, programs and work places) and personal factors (perceptions of French as a foreign language, of teaching and learning methodologies and of learners) that influence this use.

\section{INDEX}

Keywords : French as a foreign language course books, professional practice of teachers, planning, institutional social and personal ends

Mots-clés : manuels de français langue étrangère, pratiques des enseignants, planification, finalités institutionnelle sociale et personnelle

\section{AUTEUR}

\section{MARGARET BENTO}

Université Paris Descartes - Laboratoire Éducation, Discours, Apprentissages (EDA)

Margaret Bento est professeure en sciences du langage spécialisée en didactique des langues au laboratoire Éducation, Discours et Apprentissages de l'université Paris Descartes. Elle s'intéresse tout particulièrement à la transposition didactique et à la gestion des ressources par les enseignants de langues étrangères.

Courriel : margaret.bento[at]parisdescartes.fr. 